\title{
The Coherent State of the Landau Hamiltonian and the Relativistic Corrections to the Zeeman Effect in $\mathrm{He}^{+}$Ions
}

\author{
Kunnat Sebastian', Wangyao Li ${ }^{2}$ \\ ${ }^{1}$ Department of Physics and Applied Physics, University of Massachusetts Lowell, Lowell, MA \\ ${ }^{2}$ School of Physical Science and Technology, ShanghaiTech University, Shanghai, China \\ Email: Kunnat_Sebastian@uml.edu,Liwy2@shanghaitech.edu.cn
}

How to cite this paper: Sebastian, K. and Li, W.Y. (2020) The Coherent State of the Landau Hamiltonian and the Relativistic Corrections to the Zeeman Effect in $\mathrm{He}^{+}$Ions. Journal of Modern Physics, 11, 455-474.

https://doi.org/10.4236/jmp.2020.113029

Received: December 11, 2019

Accepted: March 17, 2020

Published: March 20, 2020

Copyright $\odot 2020$ by author(s) and Scientific Research Publishing Inc. This work is licensed under the Creative Commons Attribution International License (CC BY 4.0).

http://creativecommons.org/licenses/by/4.0/ (c) (i) Open Access

\begin{abstract}
We calculate the energy levels of $\mathrm{He}^{+}$ion placed in a uniform magnetic field directed perpendicular to the direction of its center of mass (CM) velocity vector, correct to relative order $\frac{v^{2}}{c^{2}}$. Our calculations are within the frame work of an approximately relativistic theory, correct to relative order $\frac{v^{2}}{c^{2}}$, of a two-particle composite system bound by electromagnetic forces, and writ-
\end{abstract} ten in terms of the position, momentum and spin operators of the constituent particles as proposed by Krajcik and Foldy, and also by Close and Osborn. Since the $\mathrm{He}^{+}$ion has a net electric charge, the total or the CM momentum is not conserved and a neat separation of the CM and the internal motion is not possible. What is new in our approach is that, for the basis states in a first order degenerate perturbation theory to calculate the effects of the external magnetic field, we use the direct product of the coherent state of the Landau Hamiltonian of the $\mathrm{He}^{+}$ion in a uniform magnetic field and of the simultaneous eigenstate of the internal Hamiltonian $h, j^{2}, l^{2}, s^{2}$ and $j_{z}$, where $\boldsymbol{j}, \boldsymbol{I}$ and $\boldsymbol{s}$ are the internal total, orbital and spin angular moments of the $\mathrm{He}^{+}$ion. The coherent state is an excellent approximation to the expected classical circular motion of the center of mass (CM) of the $\mathrm{He}^{+}$ion. In addition to the $Z^{2} \alpha^{2}$ corrections to the usual nonrelativistic results, including the small corrections due to the nuclear motion, we also obtain corrections which depend on the kinetic energy $\left(E_{C M}\right)$ of the CM circular motion of the $\mathrm{He}^{+}$ion, in a nontrivial way. Even though these corrections are proportional to $\frac{E_{C M}}{M c^{2}}$, where $M$ is the mass of the $\mathrm{He}^{+}$ion, and are small for nonrelativistic CM motion, the results should be verifiable in careful experiments. Our results may also have application in astrophysical observations of the spectral 
lines of $\mathrm{He}^{+}$ions in magnetized astrophysical objects.

\section{Keywords}

Relativistic Corrections, Zeman Effect, Helium Ion in a Magnetic Field, Coherent State, Landau Hamiltonian, Energy Levels

\section{Introduction}

When a composite system with a nonzero net electric charge is placed in a uniform magnetic field, neither the total canonical or mechanical momenta are conserved. So there is no inertial frame where the total momentum is zero at all times. So the problem of separating the c.m. motion from the internal motion for such a system will be different from that of an isolated system where the total or the c.m. momentum is conserved. In the past, several authors [1]-[8] have studied the problem of calculating the corrections to the internal energy levels of composite ions in a magnetic field. Some of them [1] [2] [3] [4] are based on a constant of motion introduced initially by Baye. And some of the works treated N-body problem [6] [8]. There are also aothors introducing new momentas using the coordinates transformation [5], however the coupling term is not small when one of the particles is much heavier than the other. In the paper [7], the the center of mass motion in an electromagnetic radiation is researched. In this paper, we take a different approach to this problem which is more physical. First of all we note that the overall or the c.m. motion of the ion in a uniform magnetic field under ordinary circumstances, is the familiar classical circular motion, if the uniform magnetic field is directed perpendicular to the plane of motion of the ion. In a recent paper [9] we have shown that the quantum state which most closely resembles the classical state of a charged particle moving in a uniform magnetic field, is the coherent state of the Landau quantum Hamiltonian of such a particle. In this paper, we will calculate the corrections to the energy levels of a $\mathrm{H}$ like ion (specifically $\mathrm{He}^{+}$ion) in a uniform magnetic field, by treating the state of the c.m. motion of the ion as a coherent state of the Landau Hamiltonian. One of the interesting features of our results is that the shifts in energy levels of the ion, including their first order relativistic corrections, depend on the energy of the c.m. motion, in a significant way. Even though these corrections are of the order of $\frac{E_{C M}}{M c^{2}}$ times the usual results where $M$ is the mass of the $\mathrm{He}^{+}$ion, and hence small for nonrelativistic c.m. motion, which we assume, it is important to include them, since in the future, more precise spectroscopic measurements may be able to detect such small corrections.

The format of the rest of the paper is as follows: In Section 2, we describe the approximately relativistic (correct to the order $\frac{v^{2}}{c^{2}}$ ) Hamiltonian of the isolated $\mathrm{He}^{+}$ion, and from there, how we obtain the Hamiltonian of the system in the 
presence of an external magnetic field. We also introduce the approximately relativistic [10] [11] relations, again correct to order $\frac{v^{2}}{c^{2}}$, between the constituent and the c.m. variables. We then express these Hamiltonians in terms of these variables. In Section 3, we give the details of our perturbative calculation of the corrections to the energy levels due to the external uniform magnetic field. We also give particular attention to the basis states used in the first order perturbation calculations. The basis states we used, are the direct product of an internal state and the c.m. state which is the coherent state of the Landau Hamiltonian of a particle of charge $(Z-1) e$ and mass $M=\left(m_{e}+m_{N}\right)$ in a uniform magnetic field. In Section 4 we give our final results. Finally in Section 5, we make some concluding remarks.

\section{Hamiltonian of the $\mathrm{He}^{+}$Ion in a Uniform Magnetic Field, Including Their First Order $\left(\frac{v^{2}}{c^{2}}\right)$ Relativistic Corrections}

\subsection{Isolated $\mathrm{He}^{+}$Ion}

First let us consider the isolated $\mathrm{He}^{+}$ion, which is a weakly bound composite system of two particles where the first particle is the electron of mass $m$ and electric charge $-e$, and spin $s=\frac{1}{2}$ and the second particle is the $\mathrm{He}^{4}$ nucleus of electric charge $Z e$ and mass $m_{N}$ with zero spin and zero magnetic moment. We will put $Z=2$ only when we do numerical calculations. The Hamiltonian of such an electromagnetically bound system can be written, correct to order $\frac{v^{2}}{c^{2}}$ using the methods of Close and Osborn [12].

$$
\begin{aligned}
H= & \frac{p_{1}^{2}}{2 m}+\frac{p_{2}^{2}}{2 m_{N}}-\frac{p_{1}^{4}}{8 m^{3} c^{2}}-\frac{p_{2}^{4}}{8 m_{N}^{3} c^{2}}-\frac{Z e^{2}}{\left|\boldsymbol{r}_{1}-\boldsymbol{r}_{2}\right|}+\frac{Z e^{2}}{4 m m_{N} c^{2}}\left[\boldsymbol{p}_{1} \frac{1}{\left|\boldsymbol{r}_{1}-\boldsymbol{r}_{2}\right|} \cdot \boldsymbol{p}_{2}\right. \\
& \left.+\boldsymbol{p}_{1} \cdot\left(\boldsymbol{r}_{1}-\boldsymbol{r}_{2}\right) \frac{1}{\left|\boldsymbol{r}_{1}-\boldsymbol{r}_{2}\right|^{3}}\left(\boldsymbol{r}_{1}-\boldsymbol{r}_{2}\right) \cdot \boldsymbol{p}_{2}+H . C .\right]-\frac{Z e^{2}}{m m_{N} c^{2}} \frac{\boldsymbol{s}_{1} \cdot\left[\left(\boldsymbol{r}_{1}-\boldsymbol{r}_{2}\right) \times \boldsymbol{p}_{2}\right]}{\left|\boldsymbol{r}_{1}-\boldsymbol{r}_{2}\right|^{3}} \\
& -\pi \frac{Z e^{2}}{4 m^{2} c^{2}} \delta\left(\boldsymbol{r}_{1}-\boldsymbol{r}_{2}\right)+\frac{Z e^{2}}{2 m^{2} c^{2}} \frac{\boldsymbol{s}_{1} \cdot\left[\left(\boldsymbol{r}_{1}-\boldsymbol{r}_{2}\right) \times \boldsymbol{p}_{1}\right]}{\left|\boldsymbol{r}_{1}-\boldsymbol{r}_{2}\right|^{3}}
\end{aligned}
$$

The first line in the above equation represents the kinetic energy terms, including their first order $\left(\frac{v^{2}}{c^{2}}\right)$ corrections and the Coulomb potential energy of the two charges $Z e$ and $-e$. The second and third lines are the straight forward Breit interaction, resulting from the unretarded transverse one photon exchange. The fourth line represents the interaction between the spin magnetic moment of the electron and the magnetic field produced by the motion of the charged nucleus. The fifth line represents the effect of the zitterbewegung of the electron's motion, coming from the Darwin term, in the nonrelativistic reduction of the Dirac Hamiltonian of the electron. The sixth line represents the conventional 
spin-orbit interaction, including the so called Thomas precession. The above expression for the Hamiltonian is also the same as the expression for the Hamiltonian of a two particle system interacting electromagnetically, given by Krajcik and Foldy [10], specialized to our case, where one of the particles $\mathrm{He}^{4}$ nucleus has zero spin and zero magnetic moment. We have also put the electronic spin magnetic moment $\mu_{e}$ in their expressions [10] [11] as $\mu_{e}=-\frac{e}{2 m c}$ which neglects the radiative corrections. This is consistent with the spirit of their approach which neglected the radiative corrections to the one photon exchange between two charged particles. It should also be noted we have used the Gaussian system of units, where as Close and Osborn [12] and Krajcik and Foldy [10] used rationalized Gaussian units. Also in Equation (1) we have used the system of units where $\hbar=1$, but $c \neq 1$, where as the previous authors [10] [11] have put $\hbar=c=1$.

It has already been demonstrated [10] [13] that the above Hamiltonian is part of a consistent relativistic theory to order $\frac{1}{c^{2}}$, by giving explicit expression [10] for the ten generators of the Poincare group in terms of the basic variables of the theory, $\boldsymbol{r}_{\mu}, \boldsymbol{p}_{\mu}$ and $\boldsymbol{s}_{\mu}(\mu=1,2)$ and showing that they satisfy the commutation relations of the Poincare group to the same $\frac{1}{c^{2}}$ order. The relativistic c.m. and internal variables are defined by the requirement that when the ten generators are expressed in terms of them, they take the single particle form [10] [11] [12] to the same order $\frac{1}{c^{2}}$.

Using this requirement, the relativistic relations between the constituent variables $\left(\boldsymbol{r}_{\mu}, \boldsymbol{p}_{\mu}\right.$ and $\left.\boldsymbol{s}_{\mu}, \mu=1,2\right)$ and the c.m. and the internal variables ( $\boldsymbol{R}$, $\boldsymbol{P}, \boldsymbol{q}, \boldsymbol{p}$ and $\left.\boldsymbol{\sigma}_{\mu},(\mu=1,2)\right)$ for a two particle composite system made up of two particles, particle 1 being the electron of mass $m$ and charge $-e$ and particle 2 being the nucleus of mass $m_{N}$ and charge $+Z e$, are given by [10] [11] (correct to order $\frac{v^{2}}{c^{2}}$ ).

$$
\begin{aligned}
\boldsymbol{r}_{1}= & \boldsymbol{R}+\frac{m_{N}}{M} \boldsymbol{q}-\frac{1}{2} \frac{\boldsymbol{q}}{c^{2}}\left[\frac{m_{N}-m}{2 m m_{N} M} p^{2}+\frac{1}{2 M^{2}} \boldsymbol{P} \cdot \boldsymbol{p}\right]+H . C . \\
& +\frac{1}{2} \boldsymbol{q} \cdot \boldsymbol{P} \frac{1}{M^{2} c^{2}}\left(\frac{1}{2} \boldsymbol{p}-\frac{m_{N}}{m} \boldsymbol{p}-\frac{m_{N}}{2 M} \boldsymbol{P}\right)+H . C . \\
& -\frac{1}{2 m M c^{2}} \boldsymbol{p} \times \boldsymbol{\sigma}_{e}+\frac{m_{N}}{2 m M^{2} c^{2}} \boldsymbol{P} \times \boldsymbol{\sigma}_{e}+\frac{Z e^{2}}{2 M^{2} c^{2}}\left(m_{N}-m\right) \frac{\boldsymbol{q}}{q} \\
\boldsymbol{r}_{2}= & \boldsymbol{R}-\frac{m}{M} \boldsymbol{q}-\frac{1}{2} \frac{\boldsymbol{q}}{c^{2}}\left[\frac{m_{N}-m}{2 m m_{N} M} p^{2}+\frac{1}{2 M^{2}} \boldsymbol{P} \cdot \boldsymbol{p}\right]+H . C . \\
& +\frac{1}{2} \boldsymbol{q} \cdot \boldsymbol{P} \frac{1}{M^{2} c^{2}}\left(\frac{1}{2} \boldsymbol{p}-\frac{m}{m_{N}} \boldsymbol{p}+\frac{m}{2 M} \boldsymbol{P}\right)+H . C . \\
& -\frac{1}{2 m M c^{2}} \boldsymbol{p} \times \boldsymbol{\sigma}_{e}-\frac{1}{2 M^{2} c^{2}} \boldsymbol{P} \times \boldsymbol{\sigma}_{e}+\frac{Z e^{2}}{2 M^{2} c^{2}}\left(m_{N}-m\right) \frac{\boldsymbol{q}}{q}
\end{aligned}
$$




$$
\begin{gathered}
\boldsymbol{p}_{1}=\boldsymbol{p}+\frac{m}{M} \boldsymbol{P}+\left[\left(\frac{m_{N}-m}{2 m m_{N}}\right) p^{2}+\frac{\boldsymbol{p} \cdot \boldsymbol{P}}{2 M}\right] \frac{\boldsymbol{P}}{M c^{2}} \\
-\frac{Z e^{2}}{2 M^{2} c^{2}}\left(m_{N}-m\right) \frac{1}{q} \boldsymbol{P}+\frac{Z e^{2}}{2 M^{2} c^{2}}\left(m_{N}-m\right)(\boldsymbol{q} \cdot \boldsymbol{P}) \frac{\boldsymbol{q}}{q^{3}} \\
\boldsymbol{p}_{2}=-\boldsymbol{p}+\frac{m_{N}}{M} \boldsymbol{P}-\left[\left(\frac{m_{N}-m}{2 m m_{N}}\right) p^{2}+\frac{\boldsymbol{p} \cdot \boldsymbol{P}}{2 M}\right] \frac{\boldsymbol{P}}{M c^{2}} \\
+\frac{Z e^{2}}{2 M^{2} c^{2}}\left(m_{N}-m\right) \frac{1}{q} \boldsymbol{P}+\frac{Z e^{2}}{2 M^{2} c^{2}}\left(m_{N}-m\right)(\boldsymbol{q} \cdot \boldsymbol{P}) \frac{\boldsymbol{q}}{q^{3}} \\
\boldsymbol{s}_{e}=\boldsymbol{\sigma}_{e}-\frac{\boldsymbol{\sigma}_{e} \times(\boldsymbol{p} \times \boldsymbol{P})}{2 m M c^{2}} \\
\boldsymbol{s}_{N}=\sigma_{N}+\frac{\boldsymbol{\sigma}_{N} \times(\boldsymbol{p} \times \boldsymbol{P})}{2 m_{N} M c^{2}}
\end{gathered}
$$

where, $\quad M=m+m_{N}$

$$
\begin{gathered}
\boldsymbol{q}=\left.\left(\boldsymbol{r}_{1}-\boldsymbol{r}_{2}\right)\right|_{\boldsymbol{P}=0} \\
\boldsymbol{p}=\left.\boldsymbol{p}_{1}\right|_{\boldsymbol{P}=0}=-\left.\boldsymbol{p}_{2}\right|_{\boldsymbol{P}=0} \\
\boldsymbol{P}=\boldsymbol{p}_{1}+\boldsymbol{p}_{2} \\
\boldsymbol{R}=\left.\frac{m \boldsymbol{r}_{1}+m_{N} \boldsymbol{r}_{2}}{M}\right|_{\boldsymbol{P}=0}
\end{gathered}
$$

We also notice, since we put $\hbar=1$,

$$
\begin{aligned}
& {\left[q_{i}, p_{j}\right]=i \delta_{i j}} \\
& {\left[R_{i}, P_{j}\right]=i \delta_{i j}}
\end{aligned}
$$

Also $\boldsymbol{R}$ and $\boldsymbol{P}$ commute with the internal variables $\boldsymbol{q}$ and $\boldsymbol{p}$ as well as $\sigma_{e}$ and $\sigma_{N}$. In our case, for the $\mathrm{He}^{4}$ nucleus, $s_{N}$ and $\sigma_{N}$ both vanish. $\sigma_{N}$ is the spin operator of the electron in the $\boldsymbol{P}=0$ frame. The reader can easily verify that when expressed in terms of the c.m. and the internal variables, the Hamiltonian of Equation (1) takes the single particle form,

$$
H=\sqrt{c^{2} P^{2}+h^{2}}
$$

to order $\frac{v^{2}}{c^{2}}$. The other nine generators also take the single particle form again to order $\frac{v^{2}}{c^{2}}$, In particular, to order $\frac{v^{2}}{c^{2}}$, Equation (11) takes the form,

$$
H=M c^{2}+h^{(0)}\left(1-\frac{P^{2}}{2 M^{2} c^{2}}\right)+h^{(1)}-\frac{P^{4}}{8 M^{3} c^{2}}
$$

where $h^{(0)}$ is the nonrelativistic internal Hamiltonian and $h^{(0)}$ is the first or$\operatorname{der}\left(\frac{v^{2}}{c^{2}}\right)$ correction to $h^{(0)}$. We find,

$$
h^{(0)}=\frac{p^{2}}{2 \mu}-\frac{Z e^{2}}{q}
$$




$$
\begin{aligned}
h^{(1)}= & -\frac{p^{4}}{8 m^{3} c^{2}}-\frac{p^{4}}{8 m_{N}^{3} c^{2}}-\frac{Z e^{2}}{4 m m_{N} c^{2}}\left(\boldsymbol{p} \frac{1}{q} \cdot \boldsymbol{p}+H . C .\right) \\
& -\frac{Z e^{2}}{4 m m_{N} c^{2}}\left(\boldsymbol{p} \cdot \boldsymbol{q} \frac{1}{q^{3}} \cdot \boldsymbol{q} \cdot \boldsymbol{p}+H . C .\right)+\frac{Z e^{2}}{m m_{N} c^{2}} \frac{\boldsymbol{\sigma}_{e} \cdot(\boldsymbol{q} \times \boldsymbol{p})}{q^{3}} \\
& -\pi \frac{Z e^{2}}{4 m^{2} c^{2}} \delta(\boldsymbol{q})+\frac{Z e^{2}}{2 m^{2} c^{2}} \frac{\boldsymbol{\sigma}_{e} \cdot(\boldsymbol{q} \times \boldsymbol{p})}{q^{3}}
\end{aligned}
$$

In Equation (16) $\mu$ is the reduced mass

$$
\mu=\frac{m m_{N}}{m+m_{N}}=\frac{m m_{N}}{M}
$$

It should be stressed that Equation (1) would not have taken the single particle form of Equation (12) if we had used the nonrelativistic c.m. variables, which means neglecting the $\frac{1}{c^{2}}$ terms in Equation (2) and Equation (3). Also we will find with the nonrelativistic c.m. variables, the center of mass itself does not move uniformly and that the internal angular momentum, $\boldsymbol{I}=\boldsymbol{q} \times \boldsymbol{p}$ is not conserved. We should emphasize that $\sigma_{e}$ in Equation (14) and elsewhere in the paper is not the Pauli matrix, but the spin $\frac{1}{2}$ operator of the electron, $\boldsymbol{s}_{e}$, in the $\boldsymbol{P}=0$ frame, which is really one half the usual Pauli matrix.

In order to derive Equations (12)-(14) we made use of the following convenient relations between the constituent and linearly independent c.m. and internal variables $\boldsymbol{R}, \boldsymbol{P}, \boldsymbol{q}$ and $\boldsymbol{p}$, which can be derived from Equations (2)-(4) using Equation (6) and Equation (7).

$$
\begin{aligned}
\boldsymbol{r}_{1}-\boldsymbol{r}_{2}= & \boldsymbol{q}-\frac{1}{2 M^{2} c^{2}}\left(\frac{m_{N}}{m}-\frac{m}{m_{N}}\right)[(\boldsymbol{q} \cdot \boldsymbol{P}) \boldsymbol{p}+\boldsymbol{p}(\boldsymbol{q} \cdot \boldsymbol{P})] \\
& -\frac{1}{2 M^{2} c^{2}}(\boldsymbol{q} \cdot \boldsymbol{P}) \boldsymbol{P}-\frac{\boldsymbol{\sigma}_{e} \times \boldsymbol{P}}{2 m M c^{2}} \\
\left|\boldsymbol{r}_{1}-\boldsymbol{r}_{2}\right|=q & {\left[1-\frac{1}{2 M^{2} c^{2}}\left(\frac{m_{N}}{m}-\frac{m}{m_{N}}\right) \times \frac{1}{q^{2}}[(\boldsymbol{q} \cdot \boldsymbol{P})(\boldsymbol{q} \cdot \boldsymbol{p})+(\boldsymbol{q} \cdot \boldsymbol{p})(\boldsymbol{q} \cdot \boldsymbol{P})]\right.} \\
& \left.-\frac{1}{2 M^{2} c^{2}} \frac{1}{q^{2}}(\boldsymbol{q} \cdot \boldsymbol{P})^{2}-\frac{\boldsymbol{q} \cdot\left(\boldsymbol{\sigma}_{e} \times \boldsymbol{P}\right)}{2 m M c^{2} q^{2}}\right]
\end{aligned}
$$

The nonrelativistic internal Hamiltonian $h^{(0)}$ of Equation (13) gives the Bohr energy levels of a particle of reduced mass $\mu$ given by Equation (15). The first order relativistic correction to this Hamiltonian, $h^{(1)}$ of Equation (14), gives the fine structure corrections to the energy level due to the spin-orbit, magnetic and Darwin terms. It is important to note our expressions for $h^{(0)}$ and $h^{(1)}$ includes the effects of nuclear motion, that is terms of all orders in $\left(\frac{m}{m_{N}}\right)$ correct to order $\frac{v^{2}}{c^{2}}$. We will calculate the corrections to the Bohr energy levels due to $h^{(1)}$ in the first order degenerate perturbation theory where the basis states are chosen as the simultaneous eigen stats of $h^{(0)}, l^{2}, \sigma_{e}^{2}, j^{2}$ and 
$j_{z}$, where,

$$
\begin{gathered}
\boldsymbol{l}=\boldsymbol{q} \times \boldsymbol{p} \\
\boldsymbol{j}=\boldsymbol{l}+\boldsymbol{\sigma}_{e}
\end{gathered}
$$

\subsection{The Hamiltonian of the $\mathrm{He}^{+}$Ion in a Uniform Magnetic Field}

The main goal of this paper is to calculate the corrections to the eigenvalues or energy levels of $h^{(0)}+h^{(1)}$ of the $\mathrm{He}^{+}$ion due to the presence of an external uniform magnetic field, the so called Zeeman effect on a composite system with a net electric charge. The Hamiltonian of $\mathrm{He}^{+}$ion in the presence of an external magnetic field $\boldsymbol{B}$ is obtained from the Hamiltonian of Equation (1) by means of two requirements. 1) The resulting Hamiltonian should be gauge invariant in the sense that the Hamiltonian should be invariant under the gauge transformations

$$
A_{\mu} \rightarrow A_{\mu}^{\prime}=A_{\mu}+\partial_{\mu} \chi
$$

where $\chi$ is an arbitrary function of $\boldsymbol{x}$ and $\boldsymbol{t}$, and $A_{\mu}$ is the four vector potential representing the external e.m. field $\boldsymbol{B}=\nabla \times \boldsymbol{A}$. In our case where there is only an external magnetic field, this requirement reduce to,

$$
\boldsymbol{A} \rightarrow \boldsymbol{A}^{\prime}=\boldsymbol{A}+\nabla \chi
$$

2) The resulting Hamiltonian should reduce to the sum of the Foldy-Wouthuysen reduced Hamiltonians (to order $\frac{v^{2}}{c^{2}}$ ) of two relativistic free particles in the absence of any internal interaction between the particles.

The first requirement can be satisfied if every momentum operator $\boldsymbol{p}_{\mu}$ in Equation (1) is replaced by $\left(\boldsymbol{p}_{\mu}-\frac{e_{\mu}}{c} \boldsymbol{A}_{\mu}\right)$ where $\mu=1,2$ is the particle label and $\boldsymbol{A}_{\mu}=\boldsymbol{A}\left(\boldsymbol{r}_{\mu}\right)$. The second requirement can be satisfied by adding two terms which depend on $\boldsymbol{B}$ and the spin operator of the electron and which are obtained from the Dirac Hamiltonian of the electron in the presence of a magnetic field by the Foldy-Wouthuysen reduction to order $\frac{v^{2}}{c^{2}}$. Using the two requirements, the Hamiltonian of the $\mathrm{He}^{+}$ion in the presence of an external uniform magnetic field to relative order $\frac{v^{2}}{c^{2}}$, is given by the following expression:

$$
\begin{aligned}
H= & \frac{\left(\boldsymbol{p}_{1}+\frac{e}{c} \boldsymbol{A}_{1}\right)^{2}}{2 m}+\frac{\left(\boldsymbol{p}_{2}-\frac{Z e}{c} \boldsymbol{A}_{2}\right)^{2}}{2 m_{N}}-\frac{Z e^{2}}{\left|\boldsymbol{r}_{1}-\boldsymbol{r}_{2}\right|}-\frac{\left(\boldsymbol{p}_{1}+\frac{e}{c} \boldsymbol{A}_{1}\right)^{2}\left(\boldsymbol{p}_{1}+\frac{e}{c} \boldsymbol{A}_{1}\right)^{2}}{8 m^{3} c^{2}} \\
& -\frac{\left(\boldsymbol{p}_{2}-\frac{Z e}{c} \boldsymbol{A}_{2}\right)^{2}\left(\boldsymbol{p}_{2}-\frac{Z e}{c} \boldsymbol{A}_{2}\right)^{2}}{8 m_{N}^{3} c^{2}}+\frac{Z e^{2}}{4 m m_{N} c^{2}}\left[\left(\boldsymbol{p}_{1}+\frac{e}{c} \boldsymbol{A}_{1}\right) \frac{1}{\left|\boldsymbol{r}_{1}-\boldsymbol{r}_{2}\right|}\left(\boldsymbol{p}_{2}-\frac{Z e}{c} \boldsymbol{A}_{2}\right)\right. \\
& \left.\left(\boldsymbol{p}_{1}+\frac{e}{c} \boldsymbol{A}_{1}\right) \cdot\left(\boldsymbol{r}_{1}-\boldsymbol{r}_{2}\right) \frac{1}{\left|\boldsymbol{r}_{1}-\boldsymbol{r}_{2}\right|^{3}} \times\left(\boldsymbol{r}_{1}-\boldsymbol{r}_{2}\right) \cdot\left(\boldsymbol{p}_{2}-\frac{Z e}{c} \boldsymbol{A}_{2}\right)+\text { H.C. }\right]
\end{aligned}
$$




$$
\begin{aligned}
& -\frac{Z e^{2}}{m m_{N} c^{2}} \frac{s_{1} \cdot\left[\left(\boldsymbol{r}_{1}-\boldsymbol{r}_{2}\right) \times\left(\boldsymbol{p}_{2}-\frac{Z e}{c} \boldsymbol{A}_{2}\right)\right]}{\left|\boldsymbol{r}_{1}-\boldsymbol{r}_{2}\right|^{3}}-\pi \frac{Z e^{2}}{4 m^{2} c^{2}} \delta\left(\boldsymbol{r}_{1}-\boldsymbol{r}_{2}\right) \\
& +\frac{Z e^{2}}{2 m^{2} c^{2}} \frac{s_{1} \cdot\left[\left(\boldsymbol{r}_{1}-\boldsymbol{r}_{2}\right) \times\left(\boldsymbol{p}_{1}+\frac{e}{c} \boldsymbol{A}_{1}\right)\right]}{\left|\boldsymbol{r}_{1}-\boldsymbol{r}_{2}\right|^{3}}+\frac{e}{m c} \boldsymbol{s}_{1} \cdot \boldsymbol{B}-\frac{e}{4 m^{3} c^{3}}\left[p_{1}^{2}, \boldsymbol{s}_{1} \cdot \boldsymbol{B}\right]_{+}
\end{aligned}
$$

The last two terms on the right hand side of Equation (25) represents the terms obtained from the second requirement mentioned above. In Equation (25) we have to keep terms up to order $\frac{1}{c^{3}}$ because of the $\frac{1}{c}$ factor in $\frac{1}{c} \boldsymbol{A}$ when $\boldsymbol{p}$ is replaced by $\left(\boldsymbol{p}-\frac{q}{c} \boldsymbol{A}\right)$ where $q$ is the electric charge of the particle.

We have reasons to believe we are on the right track with the Hamiltonian of Equation (21). In previous works, [14] we have shown that the Hamiltonian in the presence of an external radiation field obtained by means requirements (1) and (2) reproduced the relativistic invariance of the one and the two photon transition amplitudes together with the use of the relativistic c.m. variables introduced above. It has also been shown [13] [14] [15] that the same Hamiltonian would reproduce the well-known low energy theorems on Compton scattering of photons by bound composite systems.

In Equation (22),

$$
\boldsymbol{A}_{\mu}=\boldsymbol{A}\left(\boldsymbol{r}_{\mu}\right)
$$

where $\mu=1,2$ is the particle label. In the case of the external uniform magnetic field $\boldsymbol{B}$,

$$
\boldsymbol{A}_{\mu}=\frac{1}{2}\left(\boldsymbol{r}_{\mu} \times \boldsymbol{B}\right)
$$

where we have chosen the symmetric gauge to define the vector potential. If $\boldsymbol{B}$ is along the $Z$-axis, the vector potential $A$ will only have $x$ and $y$ components, so that

$$
\boldsymbol{A}_{\mu}=-\frac{1}{2} B y_{\mu} \hat{\boldsymbol{i}}+\frac{1}{2} B x_{\mu} \hat{\mathbf{j}}
$$

Substituting Equation (25) in Equation (22) and using the first order relativistic relations between the constituent and c.m. variables of Equations (2)-(4) and Equation (6) and Equation (7) in the resulting Equation (22) and after a series of simplifications and rearranging, we obtain the following expression for the Hamiltonian of $\mathrm{He}^{+}$ion in a uniform magnetic field directed along the $\mathrm{Z}$-axis.

$$
\begin{aligned}
H= & \frac{p^{2}}{2 \mu}-\frac{Z e^{2}}{q}-p^{4}\left(\frac{1}{8 m^{3} c^{2}}+\frac{1}{8 m_{N}^{3} c^{2}}\right)+\frac{Z e^{2}}{2 m^{2} c^{2}} \frac{1}{q^{3}} \boldsymbol{\sigma}_{e} \cdot \boldsymbol{I}\left(1+2 \frac{m}{m_{N}}\right) \\
& -\frac{Z e^{2}}{2 m m_{N} c^{2}}\left(\boldsymbol{p} \frac{1}{q} \cdot \boldsymbol{p}+\boldsymbol{p} \cdot \boldsymbol{q} \frac{1}{q} \boldsymbol{q} \cdot \boldsymbol{p}\right)-\pi \frac{Z e^{2}}{4 m^{2} c^{2}} \delta(\boldsymbol{q})
\end{aligned}
$$




$$
\begin{aligned}
& +\left(\frac{P_{X}^{2}}{2 M}+\frac{(Z-1)^{2} e^{2}}{8 M c^{2}} B^{2} X^{2}\right)+\left(\frac{P_{Y}^{2}}{2 M}+\frac{(Z-1)^{2} e^{2}}{8 M c^{2}} B^{2} Y^{2}\right) \\
& -\frac{(Z-1) e B}{2 M c} L_{Z}+\frac{e^{2} B^{2}}{8 c^{2}}\left(\frac{1}{m}+\frac{Z^{2}}{m_{N}}-\frac{(Z-1)^{2}}{M}\right)\left(X^{2}+Y^{2}\right) \\
& +\frac{(Z-1) e B}{2 M c} L_{Z} \frac{P^{2}}{2 M^{2} c^{2}}-\frac{P^{4}}{8 M^{3} c^{2}}+\frac{e^{2} B^{2}}{8 m c^{2}}\left(q^{2}-q_{z}^{2}\right)\left(\frac{m_{N}^{2}}{M^{2}}+Z^{2} \frac{m}{m_{N}} \frac{m^{2}}{M^{2}}\right) \\
& +\left(\frac{p^{2}}{2 \mu}-\frac{Z e^{2}}{q}\right) \frac{P^{2}}{2 M^{2} c^{2}}\left(1-2 \frac{m}{M}\right)-\frac{p^{2}}{2 \mu} \frac{m}{M} \frac{P^{2}}{M^{2} c^{2}} \\
& -\frac{p_{z}^{2}}{2 \mu} \frac{P^{2}}{2 M^{2} c^{2}}+\frac{e B}{2 m c}\left(\frac{m_{N}}{M}-Z \frac{m^{2}}{m_{N} M}\right) l_{z}+\frac{e B}{m c} \sigma_{e z} \\
& -\frac{e B}{2 m c} \frac{p^{2}}{2 m} l_{z}\left(\frac{m_{N}}{M} \frac{1}{m c^{2}}+\frac{m_{N}-m}{m_{N}} \frac{1}{M c^{2}}\right) \\
& -\frac{Z e B}{2 m_{N} c} \frac{p^{2}}{2 \mu}\left(\frac{m_{N}}{M}-\frac{m}{m_{N}}\right) l_{z} \frac{1}{M c^{2}} \\
& -\frac{e B}{2 m c} l_{z} \frac{P^{2}}{4 M^{2} c^{2}}\left(1-3 \frac{m}{M}-Z \frac{m}{m_{N}}\left(\frac{3}{2}+\frac{m}{2 M}\right)\right) \\
& -\frac{e B}{2 m c} \frac{Z e^{2}}{q} \frac{l_{Z}}{2 M c^{2}}\left(1-\frac{m_{N}-m}{M}-Z \frac{m}{m_{N}}\left(\frac{3}{2}+\frac{m}{2 M}\right)\right) \\
& -\frac{e B}{2 m c} \frac{p^{2}-p_{z}^{2}}{2 m} \frac{\sigma_{e z}}{2 M c^{2}}\left(1+Z \frac{m}{m_{N}}\right)-\frac{Z e B}{2 m_{N} c} \frac{Z e^{2}}{q^{3}}\left(q^{2}-q_{z}^{2}\right) \frac{\sigma_{e z}}{M c^{2}} \\
& -\frac{e B}{2 m c} \sigma_{e z} \frac{P^{2}}{2 M^{2} c^{2}}\left(1+\frac{m}{M}-Z \frac{m}{M}\right)-\frac{e B}{2 m c} \frac{p^{2}}{2 m} \frac{L_{Z}}{M c^{2}}\left(1-Z \frac{m^{2}}{m_{N}^{2}}\right) \\
& +\frac{e B}{2 m c} \frac{Z e^{2}}{q} \frac{L_{Z}}{2 M c^{2}}\left(1-Z \frac{m}{m_{N}}\right)
\end{aligned}
$$

where the c.m. orbital angular momentum operator,

$$
\boldsymbol{L}=\boldsymbol{R} \rtimes \boldsymbol{P},
$$

the operator $\sigma_{e}$ is the spin operator of the electron in the $\boldsymbol{P}=0$ frame, except for the factor $\hbar$ which we have put equal to 1 in all of the above equations.

Several comments are in order about Equation (26). First, we have included terms of order $B^{2}$ only in the nonrelativistic part of the Hamiltonian. In the relativistic correction terms, we have kept only terms which are linear in $B$, mainly because the contribution of the quadratic and the higher order terms in $B$ are demonstrably negligible, even for any reasonably strong values of the magnetic field. In the next section we will write the Hamiltonian of Equation (26) as the sum of an unperturbed Hamiltonian $H_{0}$ and a perturbation Hamiltonian $V$. The unperturbed Hamiltonian will contain $h^{(0)}+h^{(1)}$ plus the Landau Hamiltonian of a charged particle of electric charge $(Z-1) e$ and of mass $M$, moving in the xy plane perpendicular to the magnetic field $\boldsymbol{B}$ directed along the $\mathrm{Z}$-axis. The remaining terms on the right hand side of Equation (26) will be treated as the perturbation $V$. 


\section{Perturbative Calculation of Corrections to the Energy Levels of $\mathrm{He}^{+}$Ion Due to the External Uniform Magnetic Field}

For the first order perturbation calculation, we first write down our explicit expressions for $H_{0}$ and $V$.

$$
\begin{aligned}
H_{0}= & \left(\frac{p^{2}}{2 \mu}-\frac{Z e^{2}}{q}\right)-p^{4}\left(\frac{1}{8 m^{3} c^{2}}+\frac{1}{8 m_{N}^{3} c^{2}}\right) \\
& +\frac{Z e^{2}}{2 m^{2} c^{2}} \frac{1}{q^{3}} \sigma_{e} \cdot \boldsymbol{l}+\frac{Z e^{2}}{m m_{N} c^{2}} \frac{1}{q^{3}} \sigma_{e} \cdot \boldsymbol{l}-\pi \frac{Z e^{2}}{4 m^{2} c^{2}} \delta(\boldsymbol{q}) \\
& -\frac{Z e^{2}}{2 m m_{N} c^{2}}\left(\boldsymbol{p} \frac{1}{q} \cdot \boldsymbol{p}+\boldsymbol{p} \cdot \boldsymbol{q} \frac{1}{q} \boldsymbol{q} \cdot \boldsymbol{p}\right)+\left(\frac{P_{X}^{2}}{2 M}+\frac{(Z-1)^{2} e^{2}}{8 M c^{2}} B^{2} X^{2}\right) \\
& +\left(\frac{P_{Y}^{2}}{2 M}+\frac{(Z-1)^{2} e^{2}}{8 M c^{2}} B^{2} Y^{2}\right)-\frac{(Z-1) e B}{2 M c} L_{Z}
\end{aligned}
$$

We can write $H_{0}$ as,

$$
H_{0}=h^{(0)}+h^{(1)}+H_{0}^{L}
$$

where $h^{(0)}$ is the nonrelativistic internal Hamiltonian and $h^{(0)}$ is its first order (of order $\frac{v^{2}}{c^{2}}$ ) correction, proportional to $\frac{1}{c^{2}} \cdot H_{0}^{L}$ is the Landau Hamiltonian of a particle of mass $M$ and electric charge $(Z-1) e$.

$$
H_{0}^{L}=\frac{\left(P_{X}^{2}+P_{Y}^{2}\right)}{2 M}+\frac{(Z-1)^{2} e^{2} B^{2}}{8 M c^{2}}\left(X^{2}+Y^{2}\right)-\frac{(Z-1) e B}{2 M c} L_{Z}
$$

where $L_{Z}$ is given by Equation (27).

$$
\begin{aligned}
V= & \frac{e^{2} B^{2}}{8 c^{2}}\left(\frac{1}{m}+\frac{Z^{2}}{m_{N}}-\frac{(Z-1)^{2}}{M}\right)\left(X^{2}+Y^{2}\right)+\frac{(Z-1) e B}{2 M c} L_{Z} \frac{P^{2}}{2 M^{2} c^{2}}-\frac{P^{4}}{8 M^{3} c^{2}} \\
& +\left(\frac{p^{2}}{2 \mu}-\frac{Z e^{2}}{q}\right) \frac{P^{2}}{2 M^{2} c^{2}}\left(1-2 \frac{m}{M}\right)-\frac{p^{2}}{2 \mu} \frac{m}{M} \frac{P^{2}}{M^{2} c^{2}}-\frac{p_{z}^{2}}{2 \mu} \frac{P^{2}}{2 M^{2} c^{2}} \\
& +\frac{e^{2} B^{2}}{8 m c^{2}}\left(q^{2}-q_{z}^{2}\right)\left(\frac{m_{N}^{2}}{M^{2}}+Z^{2} \frac{m}{m_{N}} \frac{m^{2}}{M^{2}}\right)+\frac{e B}{2 m c}\left(\frac{m_{N}}{M}-Z \frac{m^{2}}{m_{N} M}\right) l_{z}+\frac{e B}{m c} \sigma_{e z} \\
& -\frac{e B}{2 m c} \frac{p^{2}}{2 m} l_{z}\left(\frac{m_{N}}{M} \frac{1}{m c^{2}}+\frac{m_{N}-m}{m_{N}} \frac{1}{M c^{2}}\right)-\frac{Z e B}{2 m_{N} c} \frac{p^{2}}{2 \mu}\left(\frac{m_{N}}{M}-\frac{m}{m_{N}}\right) l_{z} \frac{1}{M c^{2}} \\
& -\frac{e B}{2 m c} \frac{Z e^{2}}{q} \frac{l_{z}}{2 M c^{2}}\left(1-\frac{m_{N}-m}{M}-Z \frac{m}{m_{N}}\left(\frac{3}{2}+\frac{m}{2 M}\right)\right) \\
& -\frac{e B}{2 m c} \frac{p^{2}-p_{z}^{2}}{2 m} \frac{\sigma_{e z}}{2 M c^{2}}\left(1+Z \frac{m}{m_{N}}\right)-\frac{Z e B}{2 m_{N} c} \frac{Z e^{2}}{q^{3}}\left(q^{2}-q_{z}^{2}\right) \frac{\sigma_{e z}}{M c^{2}} \\
& -\frac{e B}{2 m c} l_{z} \frac{P^{2}}{4 M^{2} c^{2}}\left(1-3 \frac{m}{M}-Z \frac{m}{m_{N}}\left(\frac{3}{2}+\frac{m}{2 M}\right)\right) \\
& -\frac{e B}{2 m c} \sigma_{e z} \frac{P^{2}}{2 M^{2} c^{2}}\left(1+\frac{m}{M}-Z \frac{m}{M}\right)
\end{aligned}
$$




$$
-\frac{e B}{2 m c} \frac{p^{2}}{2 m} \frac{L_{Z}}{M c^{2}}\left(1-Z \frac{m^{2}}{m_{N}^{2}}\right)+\frac{e B}{2 m c} \frac{Z e^{2}}{q} \frac{L_{Z}}{2 M c^{2}}\left(1-Z \frac{m}{m_{N}}\right)
$$

There are three types of terms in the expression for $V$ given by Equation (31):

1) terms which depend only on the CM variables, contained as the first two lines of the right hand side of Equation (31)

2) the terms which depend only on the internal variables which are the remaining terms in Equation (31), except for the third, the fourth and the last four lines and

3) coupled terms which depend on the internal as well as the c.m. variables, namely, the third, the fourth and the last four lines of Equation (31). The fact that there are coupled terms is not surprising. When the composite system with a net electric charge is in an external magnetic field, the total momentum $\boldsymbol{P}$ is not conserved and we can not go to a frame where $\boldsymbol{P}=0$ for all times and so we do not expect the Hamiltonian to be uncoupled between internal and c.m. variables, as in the case of an isolated composite system.

For future reference, we will write the perturbation $V$ as

$$
V=V_{0}+V_{B}
$$

where $V_{0}$ is the sum of terms in $V$ which are independent of $B$ and $V_{B}$ is the sum of terms which depend on $B$.

Since the unperturbed Hamiltonian $H_{0}$ is the sum of the internal Hamiltonian $h^{(0)}+h^{(1)}$ and the Landau Hamiltonian $H_{0}^{L}$, we will take the basis states for the first order degenerate perturbation theory calculations, the direct product of the simultaneous eigenstates of $h^{(0)}+h^{(1)}, j^{2}, j_{z}, l^{2}$ and $s^{2}$, and the coherent states of the Landau Hamiltonian, described above. The coherent state of the Landau Hamiltonian is the simultaneous eigenstate of the annihilation operators $a_{+}$and $a_{-}$of the two dimensional simple harmonic oscillator [9], as defined in reference [9]. In this reference, we show that the coherent state is the best approximation to the classical state where the charged particle moves in a circle in the xy plane with the cyclotron angular frequency

$$
\omega_{c}=\frac{(Z-1) e}{2 M c}
$$

The coherent state can be represented by the ket vector $\left|\alpha_{+} \alpha_{-}\right\rangle$where the complex numbers $\alpha_{+}$and $\alpha_{-}$are the eigenvalues of the annihilation operators $a_{+}$ and $a_{-}$. The basis states for the perturbation calculations can then be written as $\left|\alpha_{+}, \alpha_{-}, n, j, m_{j}, l, s=\frac{1}{2}\right\rangle$, where the quantum number $n, j, m_{j}$ and $l$ have the usual meaning. For the coherent state to approximate [9] the classical state,

$$
\left|\alpha_{+}\right| \gg 1
$$

In the above basis, the perturbation part of the Hamiltonian, $V$, will be diagonal. The expectation value of $V$ in these basis stats will give the corrections to the energy levels of $H_{0}$, which are taken to be the sum of the eigenvalues of $h^{(0)}+h^{(1)}$ and the energy of the coherent states of the Landau Hamiltonian, 
which for all practical purposes [9] is the kinetic energy of circular motion of the $\mathrm{He}^{+}$ion in the uniform magnetic field.

The eigenvalues of the internal Hamiltonian $h^{(0)}+h^{(1)}$ are themselves calculated in the first order perturbation theory, treating $h^{(1)}$ as a perturbation to $h^{(0)}$. So in our calculation we implicitly assume that the corrections to the energy levels due to the external magnetic field, the so called Zeeman splittings, are much smaller than the fine structure splittings induced by $h^{(0)}$. This assumption is justified for any external magnetic field whose strength is such that,

$$
\mu_{B} B \ll(Z \alpha)^{4} \mu c^{2}
$$

where $\mu_{B}$ is the Bohr magneton $\left(\frac{e \hbar}{2 m c}\right)$ and $\alpha$ is the fine structure constant $\frac{e^{2}}{\hbar c}$. Numerically this condition is satisfied for any $B$ whose magnitude is less than 10 Tesla or $10^{5}$ gauss. For 10 Tesla, $\mu_{B} B \approx 5.79 \times 10^{-5} \mathrm{eV}$ whereas $(\mathrm{Z} \alpha)^{4} \mu c^{2} \approx 8.5 \times 10^{-3} \mathrm{eV}$. It is important to emphasize that the corrections to the energy levels due to $h^{(0)}$ include the effects of nuclear motion to all orders in $\frac{m}{M}$, at least to order $\frac{v^{2}}{c^{2}}$.

Some important comments are in order about the $B$ dependent terms in $V$ of Equation (32), which we label as $V_{B}$. In the expression for $V_{B}$, we only included those terms whose time averages over one cycle of revolution of the circular motion represented by the coherent states, do not vanish. We calculated the time dependent expectation value of the c.m. operators, $P_{X}, P_{Y}, P_{X} P_{Y}, P_{X}^{2}, P_{Y}^{2}, X$, $Y, X^{2}, Y^{2}$ etc. by using the following expression for the time dependent coherent state,

$$
|\Psi(t)\rangle=\mathrm{e}^{-\frac{i}{2} \omega_{c} t} D_{+}\left(\alpha_{+} \mathrm{e}^{-i \omega_{c} t}\right) D_{+}\left(\alpha_{-}\right)|0\rangle_{+} \otimes|0\rangle_{-}
$$

where $D_{+}$and $D_{-}$are unitary operators, as defined in our previous work [9]. The ket reactor $|0\rangle_{+}$and $|0\rangle_{-}$are the eigenstates of the number operators [9] $N_{+}=a_{+}^{\dagger} a_{+}$and $N_{-}=a_{-}^{\dagger} a_{-}$with zero eigenvalues. We also note that

$$
D_{+}^{\dagger}\left(\alpha_{+} \mathrm{e}^{-i \omega_{c} t}\right) a_{+} D_{+}\left(\alpha_{+} \mathrm{e}^{-i \omega_{c} t}\right)=a_{+}+\alpha_{+} \mathrm{e}^{-i \omega_{c} t}
$$

and its adjoint relation

$$
D_{+}^{\dagger}\left(\alpha_{+} \mathrm{e}^{-i \omega_{c} t}\right) a_{+}^{\dagger} D_{+}\left(\alpha_{+} \mathrm{e}^{-i \omega_{c} t}\right)=a_{+}^{\dagger}+\alpha_{+}^{*} \mathrm{e}^{+i \omega_{c} t}
$$

Using Equation (37) and Equation (38) and the expressions for $X$ and $Y$ in terms of $a_{+}$and $a_{-}$we would obtain,

$$
\begin{aligned}
& \left\langle\Psi(t)\left|X-X_{0 c}\right| \Psi(t)\right\rangle=\sqrt{\frac{2 \hbar}{M \omega_{c}}}\left|\alpha_{+}\right| \sin \left(\omega_{c} t+\delta\right) \\
& \left\langle\Psi(t)\left|Y-Y_{0 c}\right| \Psi(t)\right\rangle=\sqrt{\frac{2 \hbar}{M \omega_{c}}}\left|\alpha_{+}\right| \cos \left(\omega_{c} t+\delta\right)
\end{aligned}
$$

where $X_{0 c}$ and $Y_{0 c}$ are the $x$ and $y$ coordinates of the center of the circular 
orbit which are given by the expressions

$$
\begin{gathered}
X_{0 c}=\sqrt{\frac{2 \hbar}{M \omega_{c}}} \operatorname{Re}\left(\alpha_{-}\right) \\
Y_{0 c}=-\sqrt{\frac{2 \hbar}{M \omega_{c}}} \operatorname{Im}\left(\alpha_{-}\right) \\
\sin (\delta)=\frac{\operatorname{Re}\left(\alpha_{+}\right)}{\left|\alpha_{+}\right|} \\
\cos (\delta)=\frac{\operatorname{Im}\left(\alpha_{+}\right)}{\left|\alpha_{+}\right|}
\end{gathered}
$$

We also note that the Landau Hamiltonian $H_{0}^{L}$ of Equation (30), in terms of $a_{+}$and $a_{+}^{\dagger}$ becomes [9],

$$
H_{0}^{L}=\hbar \omega_{c}\left(a_{+}^{\dagger} a_{+}+\frac{1}{2}\right) .
$$

The expectation value of $H_{0}^{L}$ in the coherent state $\left|\alpha_{+} \alpha_{-}\right\rangle$is,

$$
E_{0}^{L}=E_{C M}=\hbar \omega_{c}\left(\left|\alpha_{+}\right|^{2}+\frac{1}{2}\right)
$$

So the energy of the coherent state depends only on $\left|\alpha_{+}\right|$and completely independent of $\alpha_{-}$. So for a given expectation value of the Landau Hamiltonian, there can be an infinite number of values for $\alpha_{-}$, corresponding to the infinite degeneracy of the Landau energy levels. Equations (41)-(44) suggest that this infinite degeneracy of the coherent state for a given $\left|\alpha_{+}\right|$, correspond to an infinite number of possible centers of the circle and the different phases of $\alpha_{+}$ representing different phases of the circular motion, for a given radius and a given energy of the circular motion. We have the freedom to choose he center of the circular orbit at the origin of the xy coordinate system and from Equation (41) and Equation (42) if follows that

$$
\alpha_{-}=0
$$

with this choice we will find,

$$
\begin{gathered}
\langle\Psi(t)|X| \Psi(t)\rangle=\sqrt{\frac{2 \hbar}{M \omega_{c}}}\left|\alpha_{+}\right| \sin \left(\omega_{c} t+\delta\right) \\
\langle\Psi(t)|Y| \Psi(t)\rangle=\sqrt{\frac{2 \hbar}{M \omega_{c}}}\left|\alpha_{+}\right| \cos \left(\omega_{c} t+\delta\right) \\
\left\langle\Psi(t)\left|X^{2}\right| \Psi(t)\right\rangle=\frac{\hbar}{M \omega_{c}}\left[1+\left|\alpha_{+}\right|^{2}\left(1+\cos \left(2 \omega_{c} t\right)\right)\right] \\
\left\langle\Psi(t)\left|Y^{2}\right| \Psi(t)\right\rangle=\frac{\hbar}{M \omega_{c}}\left[1+\left|\alpha_{+}\right|^{2}\left(1-\cos \left(2 \omega_{c} t\right)\right)\right] \\
\left\langle\Psi(t)\left|X^{2}+Y^{2}\right| \Psi(t)\right\rangle=\frac{2 \hbar}{M \omega_{c}}\left(1+\left|\alpha_{+}\right|^{2}\right)
\end{gathered}
$$




$$
\begin{gathered}
\left\langle\Psi(t)\left|P_{X}\right| \Psi(t)\right\rangle=\sqrt{\frac{M \omega_{c} \hbar}{2}}\left|\alpha_{+}\right| \cos \left(\omega_{c} t+\delta\right) \\
\left\langle\Psi(t)\left|P_{Y}\right| \Psi(t)\right\rangle=-\sqrt{\frac{M \omega_{c} \hbar}{2}}\left|\alpha_{+}\right| \sin \left(\omega_{c} t+\delta\right) \\
\left\langle\Psi(t)\left|P_{X}^{2}\right| \Psi(t)\right\rangle=\frac{M \omega_{c} \hbar}{4}\left[1+\left|\alpha_{+}\right|^{2}\left(1-\cos \left(2 \omega_{c} t\right)\right)\right] \\
\left\langle\Psi(t)\left|P_{Y}^{2}\right| \Psi(t)\right\rangle=\frac{M \omega_{c} \hbar}{4}\left[1+\left|\alpha_{+}\right|^{2}\left(1+\cos \left(2 \omega_{c} t\right)\right)\right] \\
\left\langle\Psi(t)\left|P_{X}^{2}+P_{Y}^{2}\right| \Psi(t)\right\rangle=\frac{M \omega_{c} \hbar}{2}\left(1+\left|\alpha_{+}\right|^{2}\right) \\
\left\langle\Psi(t)\left|P_{X} P_{Y}\right| \Psi(t)\right\rangle=\frac{M \omega_{c} \hbar}{4}\left[\operatorname{Re}\left(\alpha_{+}\right)^{2} \sin \left(2 \omega_{c} t\right)-\operatorname{Im}\left(\alpha_{+}\right)^{2} \cos \left(2 \omega_{c} t\right)\right]
\end{gathered}
$$

From Equation (48), Equation (49), Equation (53), Equation (54) and Equation (58) it is clear that the time averages of $P_{X}, P_{Y}, X, Y$ and $P_{X} P_{Y}$ will vanish over any cycle of circular revolution. Since the cyclotron frequency of revolution $\omega_{c}=\frac{(Z-1) e B}{M c}$ is of the order of $10^{10} \mathrm{~s}^{-1}$ even for $B$ as small as 1000 gauss, what we can observe for the CM variables of the circular motion will be time averages. This is why we neglected terms in the perturbation Hamiltonian $V_{B}$ whose time average over one cycle of revolution vanished.

\section{Results of the Perturbative Calculations in the First Order}

We now give the results of the first order perturbative results where $V$ of equation (31) is the perturbation and the basis states are the direct product of the eigenstates of $h^{(0)}+h^{(1)}$, of the Equation (28) and Equation (29) and the coherent states of the Landau Hamiltonian of Equation (30). We can write the first order results which depend only on the quantum numbers $n, l, j$ and $m_{j}$ as well as the magnetic field $B$ and $E_{C M}$, as,

$$
E_{n l j m_{j}}=E_{n l j}^{(0)}+\Delta E_{n l j m_{j}}^{(1)}(B)
$$

where $E_{n l j}^{(0)}$ are the eigenvalues of the internal Hamiltonian $h^{(0)}+h^{(1)}$ to first order in $h^{(1)}$, and $\Delta E_{n l j m_{j}}^{(1)}(B)$ are the first order corrections due to the perturbation $V$ of Equation (31) which depend on the external uniform magnetic field $B$.

$$
\begin{aligned}
E_{n l j}^{(0)}= & -Z^{2} \alpha^{2} \frac{1}{2} \mu c^{2} \frac{1}{n^{2}}-\frac{1}{2}(Z \alpha)^{4} \mu c^{2} \frac{1}{n^{3}}\left(\frac{m_{N}}{M}\right)^{2}\left(\frac{1}{j+\frac{1}{2}}-\frac{3}{4 n}\right) \\
& +\frac{1}{2}(Z \alpha)^{4} \mu c^{2} \frac{1}{n^{3}}\left(\frac{m_{N}}{M}\right)^{2} \frac{m}{M}\left(\frac{1}{j}-\frac{3}{4 n}\right) \\
& -\frac{1}{2}(Z \alpha)^{4} \mu c^{2}\left(\frac{m}{M}\right)^{3}\left[\frac{1}{n^{3}\left(l+\frac{1}{2}\right)}-\frac{3}{4 n^{4}}\right]
\end{aligned}
$$




$$
\begin{aligned}
& +\frac{1}{2}(Z \alpha)^{4} \mu c^{2} \frac{1}{n^{3}} \frac{m m_{N}}{M^{2}} \frac{1}{l\left(l+\frac{1}{2}\right)(l+1)} \\
& \times\left[j(j+1)-\frac{3}{4}-l(l+1)\right]\left(1-\delta_{l 0}\right)+\frac{1}{2}(Z \alpha)^{4} \mu c^{2} \frac{1}{n^{4}} \frac{m m_{N}}{M^{2}}\left(1-\frac{2 n}{l+\frac{1}{2}}\right) \\
& -\frac{Z e^{2}}{2 m m_{N} c^{2}} \hbar^{2}\left[2 \int_{0}^{\infty} R_{n l}(r) \frac{\mathrm{d} R_{n l}}{\mathrm{~d} r} \mathrm{~d} r+\int_{0}^{\infty} R_{n l}(r) \frac{\mathrm{d}^{2} R_{n l}}{\mathrm{~d}^{2} r} r \mathrm{~d} r\right]+E_{C M} \\
& \Delta E_{n l j m_{j}}^{(1)}\left(B, E_{C M}\right) \\
& =-\frac{1}{4} Z^{2} \alpha^{2} \frac{\mu c^{2}}{n^{2}}\left(\frac{E_{C M}}{M^{2} c^{2}}\right)\left(\frac{3}{2}-Z+\frac{l(l+1)-m_{j}^{2}-\frac{3}{4}}{(2 l-1)(2 l+3)}+\frac{2 m_{j}^{2}}{(2 l-1)(2 l+1)(2 l+3)}\right) \\
& +\frac{e^{2} B^{2}}{8 M^{2} c^{2}}\left(\frac{m_{N}^{2}}{m}+Z^{2} \frac{m^{2}}{m_{N}}\right) \frac{a_{0}^{2} n^{2}}{2 Z^{2}}\left[5 n^{2}+1-3 l(l+1)\right] \\
& \times\left(1-2 \frac{l(l+1)-m_{j}^{2}-\frac{3}{4}}{(2 l-1)(2 l+3)}-\frac{4 m_{j}^{2}}{(2 l-1)(2 l+1)(2 l+3)}\right) \\
& +\mu_{B} B\left(1-\frac{m}{M}\right)\left(1-Z \frac{m^{2}}{m_{N}^{2}}\right) \times \frac{j(j+1)+l(l+1)-\frac{3}{4}}{2 j(j+1)} m_{j} \\
& +\mu_{B} B \frac{j(j+1)+\frac{3}{4}-l(l+1)}{j(j+1)} m_{j}-\mu_{B} B Z^{2} \alpha^{2} \frac{1}{n^{2}} \frac{j(j+1)+l(l+1)-\frac{3}{4}}{4 j(j+1)} \\
& \times\left[\frac{m_{N}^{2}}{M^{2}}\left(1-\frac{m^{2}}{m_{N} M}\right)+Z \frac{m^{2}}{M^{2}}\left(\frac{m_{N}}{M}-\frac{m}{m_{N}}\right)\right] \\
& -\mu_{B} B Z^{2} \alpha^{2} \frac{1}{n^{2}}\left[2 \frac{m}{M}-\frac{m}{m_{N}}\left(\frac{3}{2}+\frac{m}{2 M}\right) Z\right] \frac{m_{N}}{M} \frac{m}{M} \frac{j(j+1)+l(l+1)-\frac{3}{4}}{4 j(j+1)} m_{j} \\
& -\mu_{B} B Z^{2} \alpha^{2} \frac{1}{n^{2}} \frac{1}{4}\left(\frac{m_{N}}{M}\right)^{2} \frac{m}{M}\left(1+Z \frac{m}{m_{N}}\right) \\
& \times\left(\frac{j(j+1)+\frac{3}{4}-l(l+1)}{2 j(j+1)}-\frac{2\left(j^{2}+j-m_{j}^{2}-1\right)}{(2 l-1)(2 l+1)(2 l+3)}\right) m_{j} \\
& -Z \mu_{B} B Z^{2} \alpha^{2} \frac{1}{n^{2}}\left(\frac{m}{M}\right)^{2}\left\{1-2\left[l(l+1)-m_{j}^{2}-\frac{3}{4}\right]\right. \\
& \left.-\frac{4 m_{j}^{2}}{(2 l-1)(2 l+1)(2 l+3)}\right\} \times \frac{j(j+1)+\frac{3}{4}-l(l+1)}{2 j(j+1)} m_{j} \\
& -\mu_{B} B\left(\frac{E_{C M}}{M c^{2}}\right)\left[1-3 \frac{m}{M}-Z \frac{m}{m_{N}}\left(\frac{3}{2}+\frac{m}{2 M}\right)\right]
\end{aligned}
$$




$$
\begin{aligned}
& \times \frac{j(j+1)+l(l+1)-\frac{3}{4}}{4 j(j+1)} m_{j}-\mu_{B} B\left(\frac{E_{C M}}{M c^{2}}\right)\left(1+\frac{m}{M}-Z \frac{m}{M}\right) \\
& \times \frac{j(j+1)+\frac{3}{4}-l(l+1)}{4 j(j+1)} m_{j}-\frac{(Z-1)}{2}\left(\frac{E_{C M}}{M c^{2}}\right) E_{C M}-\frac{5}{8}\left(\frac{E_{C M}}{M c^{2}}\right) E_{C M}
\end{aligned}
$$

Several comments are in order about Equation (60) and Equation (61). In these equations $E_{C M}$ is the energy of the circular motion of the C.M. of the $\mathrm{He}^{+}$ ion, corresponding to the coherent state of the Landau Hamiltonian of Equation (30). Its explicit expression is given by Equation (46). Since the CM motion represented by the coherent state [9] is practically classical, the eigenvalue of the annihilation operator $a_{+}$satisfies the condition,

$$
\left|\alpha_{+}\right| \gg 1
$$

which means the $\frac{1}{2}$ in Equation (46) can be neglected. So,

$$
\left|\alpha_{+}\right|^{2} \approx \frac{E_{C M}}{\hbar \omega_{c}}
$$

where $\omega_{c}$ is given by Equation (33). In obtaining Equation (61) we also made use of the relation,

$$
\begin{aligned}
\left\langle\alpha_{+} \alpha_{-}\left|L_{Z}\right| \alpha_{+} \alpha_{-}\right\rangle & =\left\langle\alpha_{+} \alpha_{-}\left|\hbar\left(a_{-}^{\dagger} a_{-}-a_{+}^{\dagger} a_{+}\right)\right| \alpha_{+} \alpha_{-}\right\rangle \\
& =\hbar\left(\left|\alpha_{-}\right|^{2}-\left|\alpha_{+}\right|^{2}\right)=-\hbar\left|\alpha_{+}\right|^{2}
\end{aligned}
$$

In obtaining the last equality in Equation (64) we have put $\alpha_{-}=0$, by choosing the center of the circular orbit at the origin of the xy plane. We also made use of the relation,

$$
\left\langle\alpha_{+} \alpha_{-}\left|L_{Z} \frac{P^{2}}{4 M^{2} c^{2}}\right| \alpha_{+} \alpha_{-}\right\rangle=\frac{M \hbar \omega_{c}}{2} \frac{\left|\alpha_{+}\right|^{4}}{4 M^{2} c^{2}}=\frac{\hbar \omega_{c}}{8} \frac{\left|\alpha_{+}\right|^{4}}{M c^{2}}=\frac{E_{C M}^{2}}{8 \hbar \omega_{c} M c^{2}}
$$

Also, in Equation (61) the symbol $\mu_{B}$ represents the Bohr magneton of the electron. In regular Gaussian units, where $\hbar \neq 1$

$$
\mu_{B}=\frac{e \hbar}{2 m c}=5.79 \times 10^{-5} \mathrm{eV} \cdot \mathrm{T}^{-1}=5.79 \times 10^{-9} \mathrm{eV} \cdot \mathrm{G}^{-1}
$$

In deriving Equation (31) and Equation (61) we included terms quadratic in $B$, only in the nonrelativistic terms, but not in the first order relativistic correction, for reasons mentioned before. There is only one term quadratic in $B$ in the expression for $E_{n l j m_{j}}^{(1)}$ of Equation (61). This term is of order $\left(\frac{\mu_{B} B}{\alpha^{2} \mu c^{2}}\right)$ compared to the dominant term for Zeeman splitting, which are the first two terms proportional to $\mu_{B} B$ in Equation (61). For a magnetic field $B$ even of the order of 10 Tesla, $\left(\frac{\mu_{B} B}{\alpha^{2} \mu c^{2}}\right)$ is of the order of $10^{-2}$, which is really small compared to the leading term.

The results of Equation (61) includes the usual nonrelativistic result for the 
Zeeman effect, including the effects of nuclear motion, plus all relativistic corrections of order $\frac{v^{2}}{c^{2}}$. The first two terms proportional to $\mu_{B} B$ in Equation (61) goes over to the usual nonrelativistic results in text books [16] if we let $\frac{m_{N}}{M} \rightarrow 1$ or equivalently $\frac{m}{M} \rightarrow 0$. The dominant relativistic correction is the first term proportional $\left(\mu_{B} B\right) Z^{2} \alpha^{2}$ in Equation (61). The other terms proportional to $\left(\mu_{B} B\right) Z^{2} \alpha^{2}$ are reduced by factors of $\left(\frac{m}{M}\right)$ or higher powers of $\left(\frac{m}{M}\right)$. What is really new in our approach is the correction to the Zeeman splitting due to the circular motion of the center of mass of the $\mathrm{He}^{+}$ion given by the terms proportional to $\left(\mu_{B} B\right)\left(\frac{E_{C M}}{M c^{2}}\right)$ in Equation (61). These corrections are really small, even smaller than the dominant $Z^{2} \alpha^{2}$ correction term, for nonrelativistic energies of the circular motion, which we have assumed in this paper. Nevertheless, they are interesting and can be checked in accurate experiments. Of course these terms will vanish if $E_{C M}=0$ or if the velocity of the $\mathrm{He}^{+}$ion is directed entirely along the direction of the applied magnetic field.

The last term in Equation (60) and the last two terms in Equation (61) which depend only on $E_{C M}$ is like a constant addition to the energies and does not affect the transition energies and hence frequencies of spectral lines and so may be neglected.

We should also make some important comments about Equation (60) which gives the fine structure splittings of the $\mathrm{He}^{+}$ion, including the effects of nuclear motion. If we let $\frac{m}{M} \rightarrow 0$ or equivalently $\frac{m_{N}}{M} \rightarrow 1$, our results will go over into the usual text book results [16]. In Equation (60), $R_{n l}(r)$ is the radial part of the eigenfunction of $h^{(0)}$, which are the well-known product of the exponential function and the associated Laguerre polynomials [16]. It can be shown,

$$
\int_{0}^{\infty} R_{n l}(r) \frac{\mathrm{d} R_{n l}}{\mathrm{~d} r} \mathrm{~d} r=-\frac{4 Z^{3}}{a_{0}^{3}} \frac{1}{n^{4}} \frac{1}{[(n+l) !]^{2}}
$$

The other integral involving the second derivative of $R_{n l}(r)$ is too long to write down. But from dimensional arguments, it is also proportional to $\frac{1}{a_{0}^{3}}$ where $a_{0}$ is the Bohr radius. So the contribution from both integral terms in Equation (60) to the energy levels is of the order of $\frac{1}{Z^{3}}\left(\frac{m}{M}\right)$ times the dominant fine structure term, which is the first term proportional to $(Z \alpha)^{4}$ in Equation (60), and hence utterly negligible, compared to it. The integral terms in Equation (60) come from the magnetic interaction between the moving electron and the magnetic field produced by the moving $\mathrm{He}^{4}$ nucleus, and it will of course vanish in the limit of the infinite mass for the nucleus.

Another important point we should make about our calculations is that we 
have chosen the Z-component of the CM momentum $\boldsymbol{P}$, namely,

$$
P_{Z}=0
$$

In the general case, when $P_{Z} \neq 0$, the $\mathrm{He}^{+}$ion is moving with a uniform velocity along the $\mathrm{Z}$-axis, since there is no external force in the $\mathrm{Z}$-direction. So the results in the general case when $P_{Z} \neq 0$, can be obtained by a Lorentz transformation to the appropriate order in $\frac{v}{C}$, of the total energy, including the rest mass energy. So the results for $P_{Z} \neq 0$ can be obtained from our expressions in Equation (60) and Equation (61) by adding the term, $\frac{P_{Z}^{2}}{2 M}$ to $E_{C M}$ on the right hand side of Equation (60) and multiplying the remaining terms in Equation (60) by $\left(1+\frac{P_{Z}^{2}}{2 M^{2} c^{2}}\right)$. We should also add $\frac{P_{Z}^{4}}{8 M^{3} c^{2}}$ to the right hand side of Equation (61) while multiplying all the term on the right hand side of Equation (61), except the last two terms involving only $E_{C M}$, by the factor $\left(1+\frac{P_{Z}^{2}}{2 M^{2} c^{2}}\right)$.

\section{Summary and Concluding Remarks}

We have calculated the order $\frac{v^{2}}{c^{2}}$ relativistic correction to the Zeeman Effect in $\mathrm{He}^{+}$ion, when its CM moves in a circular orbit under the action of a uniform magnetic field perpendicular to its plane of motion. We have assumed the weak field approximation in the sense that the splittings of energy levels due to the magnetic field are much smaller than the fine structure splittings. This is justified so long as the strength of the magnetic field is less than two Tesla or 20,000 Gauss. We also assumed the CM circular motion is approximately nonrelativistic. Even if the kinetic energy of the $\mathrm{CM}$ circular motion is $1 \mathrm{MeV}$, it is much less than the rest mass energy of the $\mathrm{He}^{+}$ion which is about $3.76 \times 10^{9} \mathrm{eV}$.

What is novel in our approach is that we have chosen the basis states, for the first order degenerate perturbation theory, to be the direct product of the coherent states $\left|\alpha_{+} \alpha_{-}\right\rangle$of the Landau Hamiltonian $H_{0}^{L}$ of Equation (30) and the internal state which is a simultaneous eigenstate of the internal nonrelativistic Hamiltonian $h^{(0)}, j^{2}, l^{2}, s^{2}$ and $j_{Z}$, namely $\left|n l j m_{j}\right\rangle$. The coherent state is an excellent approximation [9] to the classical state of circular motion, so long as the magnitude of the eigenvalue of the annihilation operator $a_{+}$of the Landau Hamiltonian $H_{0}^{L}$ is much greater than one. For example, for $E_{C M}=10 \mathrm{keV}$, $\left|\alpha_{+}\right| \approx 10^{4}$, when $E_{C M}=1 \mathrm{MeV},\left|\alpha_{+}\right| \approx 10^{5}$, and they suggest excellent classical approximations. Since the $\mathrm{He}^{+}$ion has a net electric charge the total momentum is not conserved and so we do not expect complete separation of CM and internal motion. In fact the coupling terms between $\mathrm{CM}$ and internal motion give corrections of order $\frac{E_{C M}}{M c^{2}}$ to the Bohr energy levels which should be detectable in careful spectroscopic experiments involving $\mathrm{He}^{+}$ion in a uniform magnetic 
field, even though $\frac{E_{C M}}{M c^{2}}$ is of the order of $10^{-5}$, when $E_{C M}$ is of the order of 10 $\mathrm{keV}$.

The study of Zeeman effect in $\mathrm{He}^{+}$ion is important in astrophysical situation [17] [18], where there are $\mathrm{He}^{+}$ions in magnetic fields. We have only considered $\mathrm{He}^{4}$ ion, where the $\mathrm{He}^{4}$ nucleus has zero spin and zero magnetic moment. But our treatment can be easily extended to $\mathrm{He}^{3}$ ion, where $\mathrm{He}^{3}$ nucleus has spin $\frac{1}{2}$ and a spin magnetic moment. In this case there will be extra terms in the Hamiltonian of Equation (1) and hence in Equation (22) which will give extra terms in the expressions of Equation (60) and Equation (61). Equation (60) will then include hyperfine splittings in the energy levels, due to the interaction of the electronic and the nuclear spin magnetic moments. The study of composite systems with net electric charge in a magnetic field has also received considerable attention [19] [20] in atomic and solid state physics.

\section{Conflicts of Interest}

The authors declare no conflicts of interest regarding the publication of this paper.

\section{References}

[1] Baye, D. (1982) Journal of Physics B: Atomic and Molecular Physics, 15, L795. https://doi.org/10.1088/0022-3700/15/22/003

[2] Baye, D. and Vincke, M. (1986) Journal of Physics B: Atomic and Molecular Physics, 19, 4051. https://doi.org/10.1088/0022-3700/19/24/009

[3] Avron, I.W. and Simon, B. (1978) Annals of Physics, 114, 431. https://doi.org/10.1016/0003-4916(78)90276-2

[4] Baye, D. (1983) Journal of Physics A: Mathematical and General, 16, 3207. https://doi.org/10.1088/0305-4470/16/14/016

[5] Dickinson, A.S. and Patterson, J.M. (1986) Journal of Physics A: Mathematical and General, 19, 1811. https://doi.org/10.1088/0305-4470/19/10/022

[6] Herold, H.R.H. and Wunner, G. (1981) Journal of Physics B: Atomic and Molecular Physics, 14, 751. https://doi.org/10.1088/0022-3700/14/4/022

[7] Thomas, I.L. (1971) Physical Review A, 3, 1022. https://doi.org/10.1103/PhysRevA.3.1022

[8] Johnson, J.O. and Yang, K.-H. (1983) Reviews of Modern Physics, 55, 109. https://doi.org/10.1103/RevModPhys.55.109

[9] Li, W. and Sebastian, K. (2018) European Journal of Physics, 39, Article ID: 045403. https://doi.org/10.1088/1361-6404/aab985

[10] Krajcik, R.A. and Foldy, L.L. (1974) Physical Review D, 10, 1777. https://doi.org/10.1103/PhysRevD.10.1777

[11] Sebastian, K.J. and Yum, D. (1979) Physical Review D, 19, 2509. https://doi.org/10.1103/PhysRevD.19.2509

[12] Close, F.E. and Osborn, H. (1970) Physical Review D, 2, 2127. https://doi.org/10.1103/PhysRevD.2.2127 
[13] Close, F.E. and Copley, L.A. (1970) Physical Review B, 19, 477. https://doi.org/10.1016/0550-3213(70)90362-7

[14] Sebastian, K.J. (1981) Physical Review A, 23, 2810. https://doi.org/10.1103/PhysRevA.23.2810

[15] Krajcik, R.A. and Foldy, L.L. (1970) Physical Review Letters, 24, 545. https://doi.org/10.1103/PhysRevLett.24.545

[16] Shankar, R. (1994) Principles of Quantum Mechanics. 2nd Edition, Springer, Berlin. https://doi.org/10.1007/978-1-4757-0576-8

[17] Richard, B.L., Crutcher, M., Troland, T.H. and Kazes, I. (1999) The Astrophysical Journal, 514, L121. https://doi.org/10.1086/311952

[18] Cooksy, G.A. and Saykally, R.J. (1986) The Astrophysical Journal, 305, L89. https://doi.org/10.1086/184691

[19] Euna Jo, J.H., An, K. and Lee, S. (2011) Physical Review B, 84, Article ID: 174423.

[20] van der Laan, G. and Thole, B.T. (1991) Physical Review B, 43, 13401. https://doi.org/10.1103/PhysRevB.43.13401 